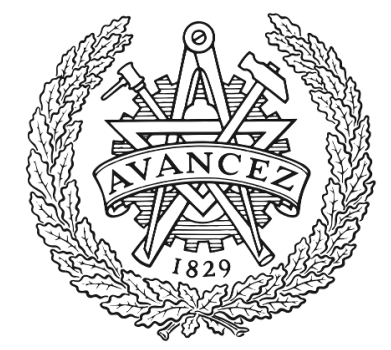

CHALMERS

UNIVERSITY OF TECHNOLOGY

\title{
Large Collective Lamb Shift of Two Distant Superconducting Artificial Atoms
}

Downloaded from: https://research.chalmers.se, 2023-04-26 04:21 UTC

Citation for the original published paper (version of record):

Wen, P., Lin, K., Frisk Kockum, A. et al (2019). Large Collective Lamb Shift of Two Distant Superconducting Artificial Atoms. Physical Review Letters, 122(23).

http://dx.doi.org/10.1103/PhysRevLett.123.233602

N.B. When citing this work, cite the original published paper. 


\title{
Large Collective Lamb Shift of Two Distant Superconducting Artificial Atoms
}

\author{
P. Y. Wen, ${ }^{1,2, *}$ K.-T. Lin,${ }^{3, *}$ A. F. Kockum, ${ }^{4,5,}{ }^{*}$ B. Suri, ${ }^{6,4}$ H. Ian, ${ }^{7,8}$ J. C. Chen, ${ }^{1,2}$ S. Y. Mao, ${ }^{9}$ \\ C. C. Chiu, ${ }^{10}$ P. Delsing, ${ }^{4}$ F. Nori $\oplus^{5,11}$ G.-D. Lin ${ }^{3}$ and I.-C. Hoi $\oplus^{1,2, \dagger}$ \\ ${ }^{1}$ Department of Physics, National Tsing Hua University, Hsinchu 30013, Taiwan \\ ${ }^{2}$ Center for Quantum Technology, National Tsing Hua University, Hsinchu 30013, Taiwan \\ ${ }^{3}$ CQSE, Department of Physics, National Taiwan University, Taipei 10617, Taiwan \\ ${ }^{4}$ Department of Microtechnology and Nanoscience, Chalmers University of Technology, 41296 Gothenburg, Sweden \\ ${ }^{5}$ Theoretical Quantum Physics Laboratory, RIKEN Cluster for Pioneering Research, Wako-shi, Saitama 351-0198, Japan \\ ${ }^{6}$ Department of Instrumentation and Applied Physics, Indian Institute of Science, Bengaluru 560012, India \\ ${ }^{7}$ Institute of Applied Physics and Materials Engineering, University of Macau, Macau, China \\ ${ }^{8}$ UMacau Zhuhai Research Institute, Zhuhai, Guangdong 519031, China \\ ${ }^{9}$ Institute of Electro-Optical Engineering, National Chiao Tung University, Hsinchu 30013, Taiwan \\ ${ }^{10}$ Department of Electrical Engineering, National Tsing Hua University, Hsinchu 30013, Taiwan \\ ${ }^{11}$ Physics Department, The University of Michigan, Ann Arbor, Michigan 48109-1040, USA
}

(Received 29 April 2019; published 4 December 2019)

\begin{abstract}
Virtual photons can mediate interaction between atoms, resulting in an energy shift known as a collective Lamb shift. Observing the collective Lamb shift is challenging, since it can be obscured by radiative decay and direct atom-atom interactions. Here, we place two superconducting qubits in a transmission line terminated by a mirror, which suppresses decay. We measure a collective Lamb shift reaching $0.8 \%$ of the qubit transition frequency and twice the transition linewidth. We also show that the qubits can interact via the transmission line even if one of them does not decay into it.
\end{abstract}

DOI: 10.1103/PhysRevLett.123.233602

Introduction.-In 1947, when attempting to pinpoint the fine structure of the hydrogen atom, Lamb and Retherford [1] discovered a small energy difference between the levels $2 S_{1 / 2}$ and $2 P_{1 / 2}$, which were thought to be degenerate according to Dirac's theory of electrons. This energy difference between the two levels can be understood when vacuum fluctuations are included in the picture, as was verified later by self-energy calculations in the framework of quantum field theory [2-4]. Briefly put, a hydrogen atom will emit photons that are instantaneously reabsorbed; while these "virtual" photons are not detectable by themselves, they leave their traces in the Lamb shift.

The hydrogen atoms that Lamb and Retherford used were obtained from a process with a very low conversion rate, so the $2 S_{1 / 2}$ level was only populated in a few atoms. Hence, the observable effects of virtual photon processes were limited to self-interaction; exchanges of virtual photons between atoms could not be detected. However, it was later realized that atom-atom interaction mediated by virtual photons also gives rise to an energy shift, referred to as a collective, or cooperative, Lamb shift (CLS) [5-9]. The atom-atom interaction also underpins the collective decay known as Dicke superradiance [10,11].

There are several obstacles impeding the experimental observation of the CLS. The shift can be enhanced by using many atoms, but, if these atoms are too close together, direct atom-atom interactions (not via virtual photons) can obscure the effect. Furthermore, the interaction giving rise to the CLS is relatively weak in three dimensions, and the shift can also be hidden by the radiative linewidth (e.g., due to the collective decay). Despite these obstacles, there have been a few experimental demonstrations of CLSs: in xenon gas [12], iron nuclei [13], rubidium vapor [14], strontium ions [15], cold rubidium atoms [16], and potassium vapor [17]. Mostly, these experiments used developments in atomic trapping and cooling [18] that have enabled higher densities of atomic ensembles, leading to a strong coupling between atomic condensates and cavity fields $[19,20]$. An improved theoretical understanding [21-23] of collective Dicke states also aided some of the experiments.

With the single exception of Ref. [15], these previous experiments all required a large number of atoms to demonstrate a CLS. The experiment of Ref. [15] only used two atoms, but the measured shift was small, $0.2 \%$ of the transition linewidth. In this Letter, we demonstrate a large CLS for two artificial atoms that exceeds the transition linewidth by a factor of 2 and reaches $0.8 \%$ of the atomic transition frequency.

Our experimental setup, depicted in Fig. 1, is a superconducting quantum circuit $[24,25]$ with two transmon qubits [26] coupled to a one-dimensional (1D) waveguide. In such superconducting circuits, strong [25,27,28], and even ultrastrong [29-31], coupling can be engineered between the qubits and photons in the waveguide. Compared to three-dimensional setups, the 1D version strengthens the interaction between qubits and reduces the 


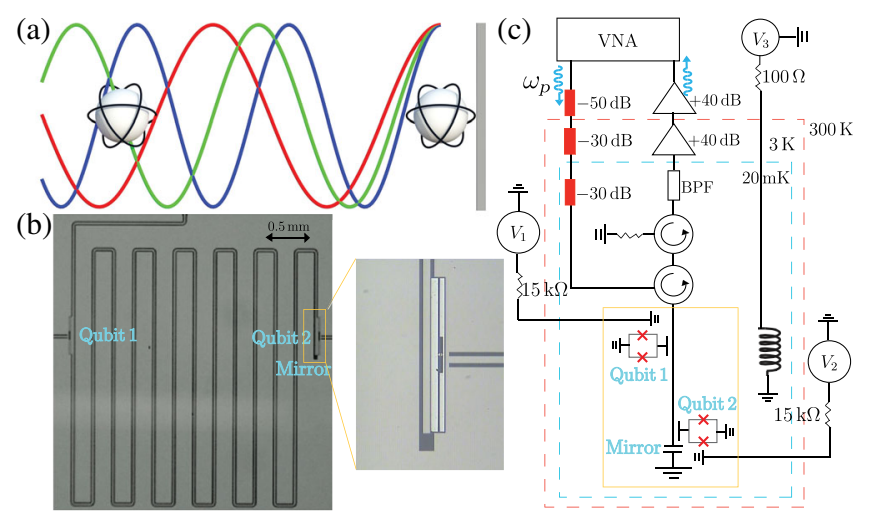

FIG. 1. Experimental setup. (a) A conceptual sketch of the setup. Two atoms are placed in front of a mirror and interact via virtual photons of different frequencies. (b) A photo of the device. Qubit 1 is placed $L \simeq 33 \mathrm{~mm}$ from Qubit 2 (enlargement on the right; the two bright parts form the qubit capacitance and the gap between them is bridged by two Josephson junctions forming a superconducting quantum interference device loop), which sits at the end of the transmission line (TL), i.e., at the mirror. The characteristic impedance of the $\mathrm{TL}$ is $Z_{0} \simeq 50 \Omega$. The relatively long distance $L$ makes it easier to tune Qubit 1 between nodes and antinodes of the electromagnetic (EM) field in the TL by tuning the qubit transition frequency. This tuning can be used to calibrate the velocity $v$ of the EM field in the TL [72]. (c) Signal routing for the experiment. Each qubit frequency can be tuned by local magnetic fields via local voltage biases $\left(V_{1}, V_{2}\right)$ and both frequencies can be tuned by a global magnetic field via $V_{3}$. For measurements, a coherent signal at frequency $\omega_{p}$ is generated by a vector network analyzer (VNA) at room temperature and fed through attenuators (red squares) to the sample, which sits in a cryostat cooled to $20 \mathrm{mK}$. At this low temperature, the number of thermal photons is negligible and the thermal excitation of the qubits should be less than a few percent [73]. The reflected signal passes a bandpass filter (BPF) and amplifiers, and is then measured with the VNA.

decay into unwanted modes. These features have enabled many important quantum-optical experiments in 1D waveguide QED in superconducting circuits in the past decade $[25,27-29,32-47]$ and inspired a wealth of theoretical studies for this platform $[25,32,48-71]$.

As shown in Fig. 1, the transmission line (TL) to which the qubits couple is terminated in a capacitive coupling to ground, which is equivalent to placing a mirror in a waveguide. The presence of this mirror separates our experiment from that of Ref. [36], where two superconducting qubits were coupled to an open TL. In such an open waveguide, the connection between collective decay and the CLS entails that the shift always will be smaller than the linewidth [59], and the measurements of elastic scattering in Ref. [36] could thus not resolve the CLS. Although a splitting in the fluorescence spectrum (inelastic scattering) indicated the presence of the CLS, it is not straightforward to extract the size of the shift from the size of the splitting $[36,59]$. In our setup, the presence of the mirror introduces interference effects that suppresses the collective decay more than the CLS [71,72,74], allowing us to clearly resolve the shift in simple reflection measurements of elastic scattering. Interestingly, it turns out that these interference effects allow us to couple the two qubits via the TL even when one of the qubits is unable to relax into the TL.

Device and characterization.-In our device, the interqubit separation $L$ is fixed. However, we can vary the qubit transition frequencies $\omega_{10}$ by applying a local magnetic flux [see Fig. 1(c)] and thus change the effective distance $L / \lambda$, where the wavelength $\lambda=2 \pi v / \omega_{10}$ and $v$ is the propagation velocity of the electromagnetic (EM) field in the waveguide [38]. Since Qubit 2 is placed next to the mirror, it will always be at an antinode of the voltage field in the waveguide [see Fig. 2(b)]. Qubit 1, on the other hand, can be tuned to a voltage node. In this case, Qubit 1 will not couple to the waveguide at its transition frequency, and thus will not contribute to any decay [38,71]. However, the CLS arises due to emission and absorption of virtual photons in all other modes of the continuum in the waveguide, which results in an interaction of strength $\Delta$ between the qubits. This interaction (CLS) leads to an avoided level crossing between the two qubits, which shows up as a frequency splitting of $2 \Delta$ in reflection measurements of the system using a weak coherent probe at frequency $\omega_{p}$.

We first characterize each of the two transmon qubits through spectroscopy. We detune the transition frequency of one of the qubits far away and measure the amplitude reflection coefficient $|r|$ of a weak coherent probe tone (i.e., the probe Rabi frequency $\Omega_{p}$ is much smaller than the decoherence rate $\gamma$ of the qubit) as a function of the flux controlling the other qubit's transition frequency and of the probe frequency $\omega_{p}$. The results are shown in Fig. 2 (Qubit 1 in the left column and Qubit 2 in the right column). For Qubit 1, which is placed at a distance $L$ from the mirror, the spectroscopy data in Fig. 2(c) show a linewidth narrowing [compare the linecuts $A$ and $B$ from Fig. 2(c), plotted in Fig. 2(e)] and a disappearing response around $4.75 \mathrm{GHz}$. At this frequency, the effective distance between Qubit 1 and the mirror is $L=7 \lambda / 4$, which places the qubit at a node for the EM field in the TL, as illustrated in Fig. 2(a), and thus effectively decouples the qubit from the TL, reducing its relaxation rate into the TL below the lowest quantity we can measure [38] $[\Gamma / 2 \pi=0.3 \mathrm{MHz}$ for $A$ in Fig. 2(e)]. Qubit 2 , on the other hand, is always at an antinode of the EM field in the TL [Fig. 2(b)] and thus has an equally strong response at all frequencies [Fig. 2(d), (f)].

We perform further spectroscopy in the full range 4-8 GHz, which is the bandwidth of the cryogenic lownoise amplifier in our experimental setup. The maximum qubit frequency is outside this bandwidth. These data are presented in the Supplemental Material [72]. From these measurements, we extract [37] the qubit relaxation rate $\Gamma$ into the TL, the pure dephasing rate $\gamma_{\phi}$ (which also contains contributions from relaxation to other channels), and the 


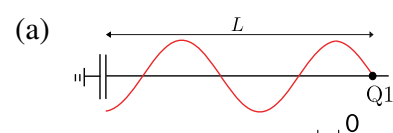

(b)

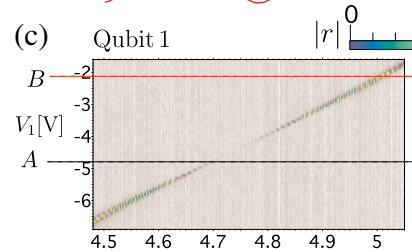

(e)
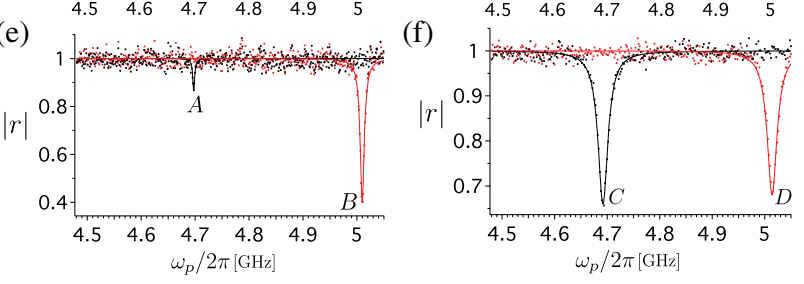

FIG. 2. Single-tone spectroscopy of the individual qubits. (a), (b) Electromagnetic mode structure (red curve) in the TL seen by Qubit 1 (Q1) and Qubit 2 (Q2), respectively. (c), (d) Amplitude reflection coefficient $|r|$ for a weak coherent probe as a function of probe frequency $\omega_{p}$ and qubit transition frequency (controlled by the voltages $V_{1}$ and $V_{3}$ for Qubit 1 and Qubit 2, respectively). Panel (c) shows how the response disappears when Qubit 1 ends up at a node for the EM field around $4.75 \mathrm{GHz}$. During these measurements, the frequency of the other qubit is tuned far from resonance with the probe. (e), (f) Linecuts from panels (c) and (d) as indicated. Dots are experimental data, and solid curves are fits following Ref. [37]. The extracted parameters are given in Table S1 in the Supplemental Material [72]. The linewidth of the dip, which occurs at the resonance $\omega_{p}=\omega_{10}$, is set by the qubit decoherence rate $\gamma=\Gamma / 2+\gamma_{\phi}$, where $\Gamma$ is the relaxation rate and $\gamma_{\phi}$ is the pure dephasing rate. Relaxation into other channels than the TL will affect the extracted value of $\gamma_{\phi}$. The depth of the dip is set by the ratio $\Gamma / \gamma_{\phi}$; since $\Gamma$ decreases close to the node of the field, the $\operatorname{dip}$ in linecut $A$ is shallower than that in $B$.

speed of light in the TL. We further use two-tone spectroscopy, driving at the qubit frequency $\omega_{10}$ and probing around the transition frequency $\omega_{21}$ from the first excited state to the second excited state, to determine the anharmonicity of the qubits. All extracted and derived parameters, for the qubits at antinodes, are summarized in Table I.

Collective Lamb shift.-We now turn to experiments where both qubits are involved and the CLS is measured. We fix the transition frequency of Qubit 2 to $\omega_{10} / 2 \pi=$ $4.75 \mathrm{GHz}$, the frequency at which Qubit 1 is at a node of the EM field [see Fig. 2(c)]. We then tune the frequency of Qubit 1 to values around this point and measure the reflection of a weak probe signal on the system for frequencies close to $\omega_{10}$. The results of these measurements are displayed in Fig. 3(a). We observe a clear anticrossing between the vertical resonance, corresponding to Qubit 2, and the diagonal resonance, corresponding to Qubit 1. The observation of this anticrossing indicates that the two qubits are coupled on resonance with strength $\Delta$ through a coherent interaction, which must be mediated by the TL since the qubits are distant from each other. The minimum size of the separation, shown in the linecut in Fig. 3(c), is $2 \Delta \simeq 2 \pi \times 38 \mathrm{MHz}$.

If the qubits were uncoupled, they would have eigenstates $|00\rangle,|01\rangle,|10\rangle$, and $|11\rangle$, with energies 0 , $\hbar \omega_{10}$, $\hbar \omega_{10}$, and $2 \hbar \omega_{10}$, respectively. Here, 0 and 1 denote ground and excited states of a single qubit, respectively; the first number in the kets is for Qubit 1 and the second number is for Qubit 2. Due to the coupling, the eigenstates $|01\rangle$ and $|10\rangle$ are replaced by the symmetric and antisymmetric eigenstates $|s\rangle=(1 / \sqrt{2})(|01\rangle+|10\rangle)$ and $|a\rangle=(1 / \sqrt{2})$ $(|01\rangle-|10\rangle)$, respectively, with eigenenergies $\hbar\left(\omega_{10} \pm \Delta\right)$ [59]. When the coupling is due to virtual photons, as in our experiment, this thus gives a CLS of $2 \hbar \Delta$, as illustrated in the inset in Fig. 3(c).

If the two qubits were placed in an open TL, it would not be possible to observe the CLS in this measurement, since each of the two resonances would have a linewidth set by a relaxation rate $\Gamma=2 \Delta$ [59]. This is not easily circumvented, since it is the coupling to the TL of the two qubits that determines both the relaxation into the TL and the strength of the interaction that is mediated via the TL. However, the presence of the mirror in our setup breaks this close connection between the linewidth and the CLS. In our setup, the CLS is given by $[71,72,74]$

$2 \Delta=\Gamma_{0}\left[\sin \left(\frac{\omega_{10}}{v}\left(x_{1}+x_{2}\right)\right)+\sin \left(\frac{\omega_{10}}{v}\left|x_{1}-x_{2}\right|\right)\right]$,

where $x_{j}$ denotes the distance of Qubit $j$ from the mirror and $\Gamma_{0}=\sqrt{\Gamma_{1}\left(\omega_{10}\right) \Gamma_{2}\left(\omega_{10}\right)}$, with $\Gamma_{j}(\omega)$ the bare relaxation rate of Qubit $j$ at frequency $\omega$ into an open TL. This is calculated using the standard master-equation approach with the Born-Markov approximation and tracing out the

TABLE I. Extracted and derived qubit parameters. We extract $\omega_{10}, \Gamma$, and $\gamma$ from fitting the spectroscopic magnitude and phase data [37]. Note that the effective relaxation rate $\Gamma$ at an antinode is twice what the relaxation rate would be in an open TL. The velocity $v$ is extracted by finding multiple nodes of the field for Qubit 1 [72]. From the two-tone spectroscopy, we extract the anharmonicity, which approximately equals the charging energy $E_{C}$ of the transmon qubits. We calculate $\gamma_{\phi}$ from $\Gamma$ and $\gamma$, and the ratio $\beta=C_{c} / C_{\Sigma}$ between the coupling capacitance $C_{c}$ to the TL and the qubit capacitance $C_{\Sigma}$ from $\Gamma$ and $E_{C}$.

\begin{tabular}{lccccccc}
\hline \hline Qubit & $E_{C} / h[\mathrm{MHz}]$ & $\omega_{10} / 2 \pi[\mathrm{GHz}]$ & $\Gamma / 2 \pi[\mathrm{MHz}]$ & $\gamma_{\phi} / 2 \pi[\mathrm{MHz}]$ & $\gamma / 2 \pi[\mathrm{MHz}]$ & $\beta$ & $v\left[10^{8} \mathrm{~m} / \mathrm{s}\right]$ \\
\hline$Q 1$ & $324 \pm 0.13$ & (Antinode) $4.068 \pm 0.00005$ & $27.18 \pm 0.24$ & $2.15 \pm 0.2$ & $15.74 \pm 0.08$ & $0.285 \pm 0.001$ & $0.8948 \pm 0.0131$ \\
$Q 2$ & $406 \pm 0.65$ & (Antinode) $4.746 \pm 0.00004$ & $28.03 \pm 0.22$ & $2.79 \pm 0.19$ & $16.8 \pm 0.08$ & $0.277 \pm 0.001$ & \\
\hline \hline
\end{tabular}



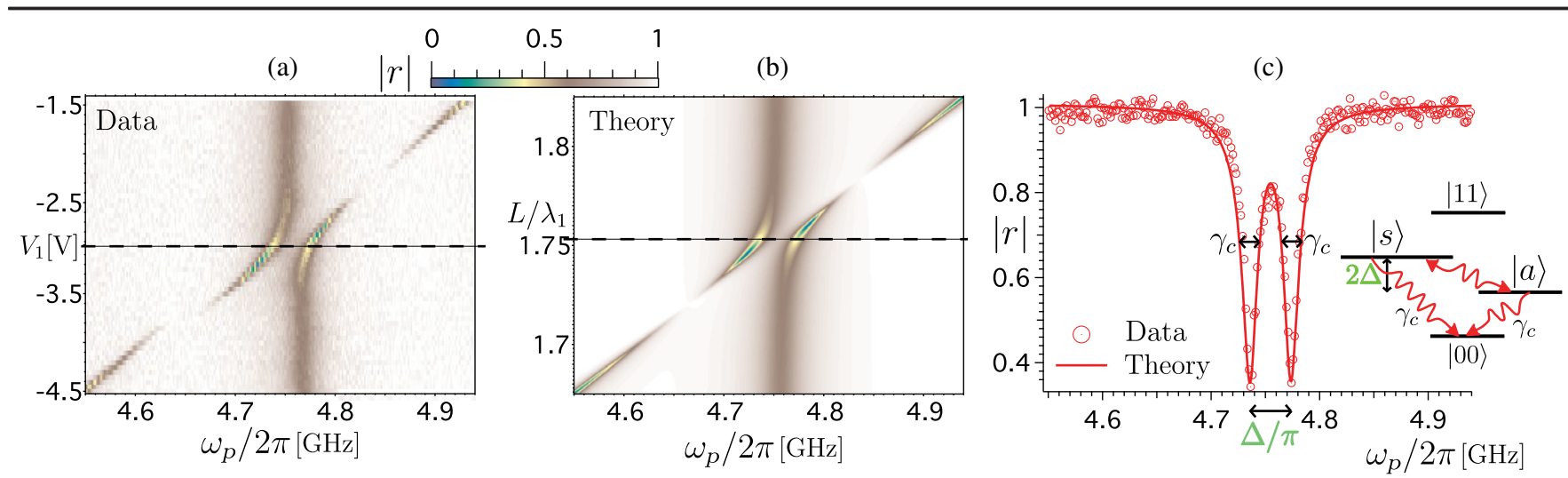

FIG. 3. Collective Lamb shift. (a) The amplitude reflection coefficient for a weak probe as a function of the probe frequency $\omega_{p}$ and the transition frequency of Qubit 1 (controlled through $V_{1}$ ). The frequency of Qubit 2 is fixed at $\omega_{10} / 2 \pi=4.75 \mathrm{GHz}$ and the frequency of Qubit 1 is tuned through resonance with this frequency. (b) Theory simulation [72] of the single-tone spectroscopy data in panel (a). The simulation is done with previously fitted parameters from Table I, with the exceptions of the free parameters $\beta_{1}=0.323$, $\beta_{2}=0.306$, and $\gamma_{\phi} / 2 \pi=2.3 \mathrm{MHz}$ for Qubit 1, which all are close to the values in Table I. The agreement between the data in (a) and the simulation in (b) is excellent. We note that the extinction of the signal around $\omega_{p} / 2 \pi=4.65 \mathrm{GHz}$ and $\omega_{p} / 2 \pi=4.85 \mathrm{GHz}$ can be explained by an interplay between detuning and decoherence [72]. (c) A linecut of the data and theory [dashed line in panels (a) and (b)], at the point where the two qubits are on resonance and the CLS $2 \Delta$ is most clearly visible. From this figure, we extract a CLS of $2 \Delta \simeq 2 \pi \times 38 \mathrm{MHz}$. The inset shows the level structure of the eigenstates of the qubits that are coupled through the CLS. The two dips in the reflection correspond to the symmetric and antisymmetric eigenstates $|s\rangle$ and $|a\rangle$. Each of these two states have the same decay rate, giving a linewidth $\gamma_{c}$ smaller than the CLS due to the presence of the mirror, as explained in the text.

photonic modes of the TL $[72,75]$. When $x_{2}=0$ and $x_{1}$ corresponds to Qubit 1 being at a node of the field in the TL, as in Fig. 3, the CLS becomes $2 \Delta=2 \Gamma_{0}$. However, since Qubit 1 is at a node, both the effective relaxation rate of Qubit 1 and the collective decay rate of the two qubits becomes zero. The only contribution from relaxation to the linewidths for the states $|s\rangle$ and $|a\rangle$ is half of $2 \Gamma_{2}$, the effective relaxation rate of Qubit 2. In this experiment, we used $\Gamma_{1} \approx \Gamma_{2}$ (giving a shift $2 \Delta \approx 2 \Gamma_{2}$ and a linewidth $\gamma_{c} \approx \Gamma_{2}$ ), but we note that the CLS could be made many
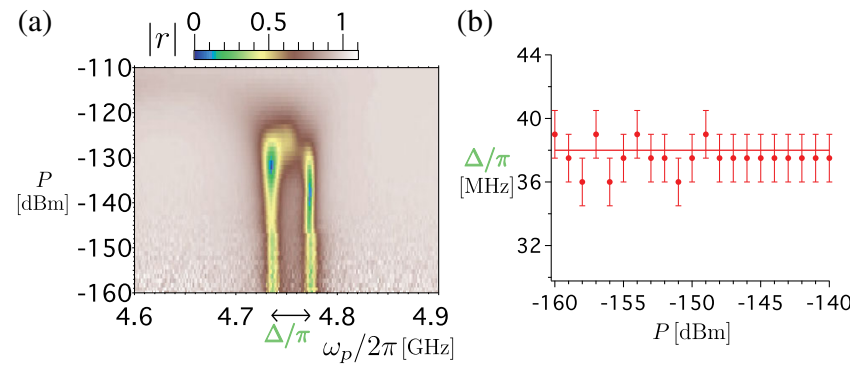

FIG. 4. Energy shift as a function of input power. (a) Amplitude reflection coefficient $|r|$ as a function of probe frequency $\omega_{p}$ and probe power $P$ for both qubit frequencies fixed at $\omega_{10} / 2 \pi=$ 4.75 GHz. The data agree very well with theoretical simulations [72]. At high probe powers, the qubits are saturated and most photons are simply reflected from the mirror, resulting in $|r| \approx 1$. (b) The extracted splitting $2 \Delta$ (red points) from panel (a) as a function of $P$, and the splitting extracted from theoretical simulations (red curve) [72]. The splitting is clearly independent of the input power in this wide range. times larger than the linewidths by instead designing the qubits such that $\Gamma_{1} \gg \Gamma_{2}$.

The fact that we can measure the CLS even though Qubit 1 ostensibly is decoupled from the TL confirms several predictions about how virtual photons influence relaxation and qubit-qubit interaction. The relaxation from Qubit 1 is stimulated by virtual photons in the TL at the transition frequency $\omega_{10}$. The relaxation is suppressed when Qubit 1 is placed at a node for the virtual photons at this frequency [38]. However, Qubit 1 is clearly coupled via virtual photons to Qubit 2. Thus, the virtual photons mediating this coupling, and causing the CLS, must have frequencies that are not equal to $\omega_{10}$. In fact, the coupling is given by a sum over all virtual modes at frequencies separate from $\omega_{10}$ [59].

Finally, we note that there are several processes, with real photons, where a strong drive shifts or dresses energy levels of qubits to create an effect that could look similar to what we have observed. To rule out such effects, e.g., the Mollow triplet [76] and Autler-Townes splitting [77], we measure $\Delta$ as a function of the power $P$ of the coherent probe. The results are shown in Fig. 4. Clearly, the energy shift $\Delta$ is independent of $P$ (before the power is high enough to saturate the qubits), indicating that the CLS we measure really is due to virtual photons.

Summary and outlook.-In this Letter, we demonstrated a large collective Lamb shift with two distant superconducting qubits in front of an effective mirror in a 1D transmission-line waveguide. Using interference effects due to the mirror, we overcame previous limitations on the size of the shift compared to the linewidth, allowing us 
to observe a shift reaching $0.8 \%$ of the qubit transition frequency and exceeding the transition linewidth. We explained how future experiments could increase the shift relative to the linewidth even more. This experiment also demonstrated that a qubit can couple to, and become entangled with, another, spatially distant qubit via the transmission line even though the first qubit is prevented from decaying into the transmission line. These results give further insight into how virtual photons affect both atomic relaxation rates and interatomic coupling, which is of fundamental importance for quantum physics, and how these effects can be controlled using interference, which could have applications in quantum communication and quantum information processing.

I.-C. H. and J. C. C. would like to thank I. A. Yu and C.-Y. Mou for fruitful discussions. I.-C. H. would like to thank Y. Lu for his help on sample fabrication. This work was financially supported by the Center for Quantum Technology from the Featured Areas Research Center Program within the framework of the Higher Education Sprout Project by the Ministry of Education (MOE) in Taiwan. I.-C. H. acknowledges financial support from the MOST of Taiwan under Projects No. 107-2112-M-007008-MY3 and No. 109-2636-M-007-007. B. S., A. F. K., and P. D. acknowledge support from the Knut and Alice Wallenberg Foundation. G.-D. L. acknowledges support from the MOST of Taiwan under Grant No. 105-2112-M002-015-MY3 and National Taiwan University under Grant No. NTUCC-108L893206. H. I. acknowledges the support by FDCT of Macau under Grant No. 065/2016/A2, by University of Macau under Grant No. MYRG2018-00088IAPME, and by NNSFC under Grant No. 11404415. J.C.C. acknowledges financial support from the MOST of Taiwan under Project No. 107-2112-M-007-003-MY3. F. N. acknowledges support from the MURI Center for Dynamic Magneto-Optics via the Air Force Office of Scientific Research (AFOSR) Grant No. FA9550-14-10040, the Army Research Office (ARO) under Grant No. W911NF-18-1-0358, the Asian Office of Aerospace Research and Development (AOARD) Grant No. FA238618-1-4045, the Japan Science and Technology Agency (JST) through the Q-LEAP program and CREST Grant No. JPMJCR1676, the Japan Society for the Promotion of Science (JSPS) through the JSPS-RFBR Grant No. 17-5250023 and the JSPS-FWO Grant No. VS.059.18N, the RIKEN-AIST Challenge Research Fund, and the NTT Physics \& Informatics Labs.

*These authors contributed equally. ichoi@phys.nthu.edu.tw

[1] W. E. Lamb and R. C. Retherford, Fine structure of the hydrogen atom by a microwave method, Phys. Rev. 72, 241 (1947).
[2] H. A. Bethe, The electromagnetic shift of energy levels, Phys. Rev. 72, 339 (1947).

[3] T. A. Welton, Some observable effects of the quantummechanical fluctuations of the electromagnetic field, Phys. Rev. 74, 1157 (1948).

[4] C. Cohen-Tannoudji, Fluctuations in radiative processes, Phys. Scr. T12, 19 (1986).

[5] V.M. Fain, On the theory of the coherent spontaneous emission, Zh. Eksp. Teor. Fiz. 36, 798 (1959) [Sov. Phys. JETP 9, 562 (1959)].

[6] R. H. Lehmberg, Radiation from an N-atom aystem. I. General formalism, Phys. Rev. A 2, 883 (1970).

[7] F. T. Arecchi and D. M. Kim, Line shifts in cooperative spontaneous emission, Opt. Commun. 2, 324 (1970).

[8] R. Friedberg, S. R. Hartmann, and J. T. Manassah, Frequency shifts in emission and absorption by resonant systems of two-level atoms, Phys. Rep. 7, 101 (1973).

[9] M. O. Scully and A. A. Svidzinsky, The Lamb shift-Yesterday, Today, and Tomorrow, Science 328, 1239 (2010).

[10] R. H. Dicke, Coherence in spontaneous radiation processes, Phys. Rev. 93, 99 (1954).

[11] N. Shammah, S. Ahmed, N. Lambert, S. De Liberato, and F. Nori, Open quantum systems with local and collective incoherent processes: Efficient numerical simulations using permutational invariance, Phys. Rev. A 98, 063815 (2018).

[12] W. R. Garrett, R. C. Hart, J. E. Wray, I. Datskou, and M. G. Payne, Large Multiple Collective Line Shifts Observed in Three-Photon Excitations of Xe, Phys. Rev. Lett. 64, 1717 (1990).

[13] R. Röhlsberger, K. Schlage, B. Sahoo, S. Couet, and R. Rüffer, Collective Lamb shift in single-photon superradiance, Science 328, 1248 (2010).

[14] J. Keaveney, A. Sargsyan, U. Krohn, I. G. Hughes, D. Sarkisyan, and C. S. Adams, Cooperative Lamb Shift in an Atomic Vapor Layer of Nanometer Thickness, Phys. Rev. Lett. 108, 173601 (2012).

[15] Z. Meir, O. Schwartz, E. Shahmoon, D. Oron, and R. Ozeri, Cooperative Lamb Shift in a Mesoscopic Atomic Array, Phys. Rev. Lett. 113, 193002 (2014).

[16] S. J. Roof, K. J. Kemp, M. D. Havey, and I. M. Sokolov, Observation of Single-Photon Superradiance and the Cooperative Lamb Shift in an Extended Sample of Cold Atoms, Phys. Rev. Lett. 117, 073003 (2016).

[17] T. Peyrot, Y. R. P. Sortais, A. Browaeys, A. Sargsyan, D. Sarkisyan, J. Keaveney, I. G. Hughes, and C.S. Adams, Collective Lamb Shift of a Nanoscale Atomic Vapor Layer within a Sapphire Cavity, Phys. Rev. Lett. 120, 243401 (2018).

[18] M. D. Lukin, Colloquium: Trapping and manipulating photon states in atomic ensembles, Rev. Mod. Phys. 75, 457 (2003).

[19] F. Brennecke, T. Donner, S. Ritter, T. Bourdel, M. Köhl, and T. Esslinger, Cavity QED with a Bose-Einstein condensate, Nature (London) 450, 268 (2007).

[20] Y. Colombe, T. Steinmetz, G. Dubois, F. Linke, D. Hunger, and J. Reichel, Strong atom-field coupling for Bose-Einstein condensates in an optical cavity on a chip, Nature (London) 450, 272 (2007).

[21] M. O. Scully, E. S. Fry, C. H. R. Ooi, and K. Wódkiewicz, Directed Spontaneous Emission from an Extended Ensemble of N Atoms: Timing is Everything, Phys. Rev. Lett. 96, 010501 (2006). 
[22] A. Svidzinsky and J.-T. Chang, Cooperative spontaneous emission as a many-body eigenvalue problem, Phys. Rev. A 77, 043833 (2008).

[23] M. O. Scully, Collective Lamb Shift in Single Photon Dicke Superradiance, Phys. Rev. Lett. 102, 143601 (2009).

[24] J. Q. You and F. Nori, Atomic physics and quantum optics using superconducting circuits, Nature (London) 474, 589 (2011).

[25] X. Gu, A. F. Kockum, A. Miranowicz, Y.-X. Liu, and F. Nori, Microwave photonics with superconducting quantum circuits, Phys. Rep. 718-719, 1 (2017).

[26] J. Koch, T. M. Yu, J. Gambetta, A. A. Houck, D. I. Schuster, J. Majer, A. Blais, M. H. Devoret, S. M. Girvin, and R. J. Schoelkopf, Charge-insensitive qubit design derived from the Cooper pair box, Phys. Rev. A 76, 042319 (2007).

[27] O. Astafiev, A. M. Zagoskin, A. A. Abdumalikov, Y. A. Pashkin, T. Yamamoto, K. Inomata, Y. Nakamura, and J. S. Tsai, Resonance fluorescence of a single artificial atom, Science 327, 840 (2010).

[28] I.-C. Hoi, C. M. Wilson, G. Johansson, T. Palomaki, B. Peropadre, and P. Delsing, Demonstration of a SinglePhoton Router in the Microwave Regime, Phys. Rev. Lett. 107, 073601 (2011).

[29] P. Forn-Díaz, J. J. García-Ripoll, B. Peropadre, J.-L. Orgiazzi, M. A. Yurtalan, R. Belyansky, C. M. Wilson, and A. Lupascu, Ultrastrong coupling of a single artificial atom to an electromagnetic continuum in the nonperturbative regime, Nat. Phys. 13, 39 (2017).

[30] L. Magazzù, P. Forn-Díaz, R. Belyansky, J.-L. Orgiazzi, M. A. Yurtalan, M. R. Otto, A. Lupascu, C. M. Wilson, and M. Grifoni, Probing the strongly driven spin-boson model in a superconducting quantum circuit, Nat. Commun. 9, 1403 (2018).

[31] A. F. Kockum, A. Miranowicz, S. De Liberato, S. Savasta, and F. Nori, Ultrastrong coupling between light and matter, Nat. Rev. Phys. 1, 19 (2019).

[32] D. Roy, C. M. Wilsonand O. Firstenberg, Colloquium: Strongly interacting photons in one-dimensional continuum, Rev. Mod. Phys. 89, 021001 (2017).

[33] O. V. Astafiev, A. A. Abdumalikov, A. M. Zagoskin, Y. A. Pashkin, Y. Nakamura, and J. S. Tsai, Ultimate On-Chip Quantum Amplifier, Phys. Rev. Lett. 104, 183603 (2010).

[34] A. A. Abdumalikov, O. V. Astafiev, Y. A. Pashkin, Y. Nakamura, and J.S. Tsai, Dynamics of Coherent and Incoherent Emission from an Artificial Atom in a 1D Space, Phys. Rev. Lett. 107, 043604 (2011).

[35] I.-C. Hoi, T. Palomaki, J. Lindkvist, G. Johansson, P. Delsing, and C.M. Wilson, Generation of Nonclassical Microwave States Using an Artificial Atom in 1D Open Space, Phys. Rev. Lett. 108, 263601 (2012).

[36] A. F. van Loo, A. Fedorov, K. Lalumiere, B. C. Sanders, A. Blais, and A. Wallraff, Photon-mediated interactions between distant artificial atoms, Science 342, 1494 (2013).

[37] I.-C. Hoi, A. F. Kockum, T. Palomaki, T. M. Stace, B. Fan, L. Tornberg, S. R. Sathyamoorthy, G. Johansson, P. Delsing, and C. M. Wilson, Giant Cross-Kerr Effect for Propagating Microwaves Induced by an Artificial Atom, Phys. Rev. Lett. 111, 053601 (2013).

[38] I.-C. Hoi, A. F. Kockum, L. Tornberg, A. Pourkabirian, G. Johansson, P. Delsing, and C. M. Wilson, Probing the quantum vacuum with an artificial atom in front of a mirror, Nat. Phys. 11, 1045 (2015).

[39] Z. H. Peng, S. E. de Graaf, J. S. Tsai, and O. V. Astafiev, Tuneable on-demand single-photon source in the microwave range, Nat. Commun. 7, 12588 (2016).

[40] Y. Liu and A. A. Houck, Quantum electrodynamics near a photonic bandgap, Nat. Phys. 13, 48 (2017).

[41] A. Yu. Dmitriev, R. Shaikhaidarov, V. N. Antonov, T. Hönigl-Decrinis, and O. V. Astafiev, Quantum wave mixing and visualisation of coherent and superposed photonic states in a waveguide, Nat. Commun. 8, 1352 (2017).

[42] P. Forn-Díaz, C. W. Warren, C. W. S. Chang, A. M. Vadiraj, and C.M. Wilson, On-Demand Microwave Generator of Shaped Single Photons, Phys. Rev. Applied 8, 054015 (2017).

[43] P. Y. Wen, A. F. Kockum, H. Ian, J. C. Chen, F. Nori, and I.-C. Hoi, Reflective Amplification without Population Inversion from a Strongly Driven Superconducting Qubit, Phys. Rev. Lett. 120, 063603 (2018).

[44] M. Mirhosseini, E. Kim, V. S. Ferreira, M. Kalaee, A. Sipahigil, A. J. Keller, and O. Painter, Superconducting metamaterials for waveguide quantum electrodynamics, Nat. Commun. 9, 3706 (2018).

[45] A. R. Hamann, C. Müller, M. Jerger, M. Zanner, J. Combes, M. Pletyukhov, M. Weides, T. M. Stace, and A. Fedorov, Nonreciprocity Realized with Quantum Nonlinearity, Phys. Rev. Lett. 121, 123601 (2018).

[46] N. M. Sundaresan, R. Lundgren, G. Zhu, A. V. Gorshkov, and A. A. Houck, Interacting Qubit-Photon Bound States with Superconducting Circuits, Phys. Rev. X 9, 011021 (2019).

[47] M. Mirhosseini, E. Kim, X. Zhang, A. Sipahigil, P. B. Dieterle, A. J. Keller, A. Asenjo-Garcia, D. E. Chang, and O. Painter, Cavity quantum electrodynamics with atom-like mirrors, Nature (London) 569, 692 (2019).

[48] J.-T. Shen and S. Fan, Coherent Single Photon Transport in a One-Dimensional Waveguide Coupled with Superconducting Quantum Bits, Phys. Rev. Lett. 95, 213001 (2005).

[49] D. E. Chang, A. S. Sørensen, E. A. Demler, and M. D. Lukin, A single-photon transistor using nanoscale surface plasmons, Nat. Phys. 3, 807 (2007).

[50] L. Zhou, Z. R. Gong, Y.-X. Liu, C. P. Sun, and F. Nori, Controllable Scattering of a Single Photon inside a OneDimensional Resonator Waveguide, Phys. Rev. Lett. 101, 100501 (2008).

[51] H. Dong, Z. R. Gong, H. Ian, L. Zhou, and C. P. Sun, Intrinsic cavity QED and emergent quasinormal modes for a single photon, Phys. Rev. A 79, 063847 (2009).

[52] H. Zheng, D. J. Gauthier, and H. U. Baranger, Waveguide QED: Many-body bound-state effects in coherent and Fockstate scattering from a two-level system, Phys. Rev. A 82, 063816 (2010).

[53] G.-Y. Chen, N. Lambert, C.-H. Chou, Y.-N. Chen, and F. Nori, Surface plasmons in a metal nanowire coupled to colloidal quantum dots: Scattering properties and quantum entanglement, Phys. Rev. B 84, 045310 (2011).

[54] A. Gonzalez-Tudela, D. Martin-Cano, E. Moreno, L. MartinMoreno, C. Tejedor, and F. J. Garcia-Vidal, Entanglement of Two Qubits Mediated by One-Dimensional Plasmonic Waveguides, Phys. Rev. Lett. 106, 020501 (2011). 
[55] D. E. Chang, L. Jiang, A. V. Gorshkov, and H. J. Kimble, Cavity QED with atomic mirrors, New J. Phys. 14, 063003 (2012).

[56] K. Koshino and Y. Nakamura, Control of the radiative level shift and linewidth of a superconducting artificial atom through a variable boundary condition, New J. Phys. 14, 043005 (2012).

[57] K. Stannigel, P. Rabl, and P. Zoller, Driven-dissipative preparation of entangled states in cascaded quantum-optical networks, New J. Phys. 14, 063014 (2012).

[58] B. Fan, A. F. Kockum, J. Combes, G. Johansson, I.-C. Hoi, C. M. Wilson, P. Delsing, G. J. Milburn, and T. M. Stace, Breakdown of the Cross-Kerr Scheme for Photon Counting, Phys. Rev. Lett. 110, 053601 (2013).

[59] K. Lalumière, B. C. Sanders, A. F. van Loo, A. Fedorov, A. Wallraff, and A. Blais, Input-output theory for waveguide QED with an ensemble of inhomogeneous atoms, Phys. Rev. A 88, 043806 (2013).

[60] B. Peropadre, J. Lindkvist, I.-C. Hoi, C. M. Wilson, J. J. Garcia-Ripoll, P. Delsing, and G. Johansson, Scattering of coherent states on a single artificial atom, New J. Phys. 15, 035009 (2013).

[61] T. Tufarelli, F. Ciccarello, and M. S. Kim, Dynamics of spontaneous emission in a single-end photonic waveguide, Phys. Rev. A 87, 013820 (2013).

[62] M. Laakso and M. Pletyukhov, Scattering of Two Photons from Two Distant Qubits: Exact Solution, Phys. Rev. Lett. 113, 183601 (2014).

[63] J. Lindkvist and G. Johansson, Scattering of coherent pulses on a two-level system-single-photon generation, New J. Phys. 16, 055018 (2014).

[64] S. R. Sathyamoorthy, L. Tornberg, A. F. Kockum, B. Q. Baragiola, J. Combes, C. M. Wilson, T. M. Stace, and G. Johansson, Quantum Nondemolition Detection of a Propagating Microwave Photon, Phys. Rev. Lett. 112, 093601 (2014).

[65] E. Shahmoon, I. Mazets, and G. Kurizki, Giant vacuum forces via transmission lines, Proc. Natl. Acad. Sci. U.S.A. 111, 10485 (2014).

[66] Y.-L. L. Fang and H. U. Baranger, Waveguide QED: Power spectra and correlations of two photons scattered off multiple distant qubits and a mirror, Phys. Rev. A 91, 053845 (2015).

[67] V. Paulisch, H. J. Kimble, and A. González-Tudela, Universal quantum computation in waveguide QED using decoherence free subspaces, New J. Phys. 18, 043041 (2016).

[68] H. Pichler and P. Zoller, Photonic Circuits with Time Delays and Quantum Feedback, Phys. Rev. Lett. 116, 093601 (2016).

[69] A. González-Tudela, V. Paulisch, H. J. Kimble, and J. I. Cirac, Efficient Multiphoton Generation in Waveguide Quantum Electrodynamics, Phys. Rev. Lett. 118, 213601 (2017).

[70] L. Guo, A. L. Grimsmo, A. F. Kockum, M. Pletyukhov, and G. Johansson, Giant acoustic atom: A single quantum system with a deterministic time delay, Phys. Rev. A 95, 053821 (2017).

[71] A. F. Kockum, G. Johansson, and F. Nori, DecoherenceFree Interaction between Giant Atoms in Waveguide Quantum Electrodynamics, Phys. Rev. Lett. 120, 140404 (2018).

[72] See Supplemental Material at http://link.aps.org/ supplemental/10.1103/PhysRevLett.123.233602 for details on the derivation of the master equation, qubit-qubit interaction, additional data from full spectroscopy, and figures for the collective Lamb shift away from the node.

[73] F. Yan, D. Campbell, P. Krantz, M. Kjaergaard, D. Kim, J. L. Yoder, D. Hover, A. Sears, A. J. Kerman, T. P. Orlando, S. Gustavsson, and W. D. Oliver, Distinguishing Coherent and Thermal Photon Noise in a Circuit Quantum Electrodynamical System, Phys. Rev. Lett. 120, 260504 (2018).

[74] K.-T. Lin, T. Hsu, C.-Y. Lee, I.-C. Hoi, and G.-D. Lin, Scalable collective Lamb shift of a 1D superconducting qubit array in front of a mirror, arXiv:1905.04743.

[75] H. J. Carmichael, Statistical Methods in Quantum Optics 1 (Springer, New York, 1999).

[76] B. R. Mollow, Power spectrum of light scattered by twolevel systems, Phys. Rev. 188, 1969 (1969).

[77] S. H. Autler and C. H. Townes, Stark effect in rapidly varying fields, Phys. Rev. 100, 703 (1955). 